\title{
Plantas medicinais utilizadas por idosos com diagnóstico de Diabetes mellitus no tratamento dos sintomas da doença
}

\author{
FEIJÓ, A.M. ${ }^{1 *}$; BUENO, M.E.N. ${ }^{1}$; CEOLIN, T. ${ }^{1}$; LINCK, C.L. ${ }^{1}$; SCHWARTZ, E. ${ }^{1}$; LANGE, C. ${ }^{1}$; MEINCKE, S.M.K. ${ }^{1}$; \\ HECK, R.M. ${ }^{1}$; BARBIERI, R.L. ${ }^{2}$; HEIDEN, G. ${ }^{2}$ \\ ${ }^{1}$ Programa de Pós-Graduação em Enfermagem da Universidade Federal de Pelotas (UFPel), Rua Gomes Carneiro, \\ 01, CEP: 96010-610, Pelotas-Brasil “aline_feijo@yahoo.com.br ²Embrapa Clima Temperado, Rodovia BR 392, \\ Km 78, Caixa Postal 403, CEP: 96001-970, Pelotas-Brasil
}

\begin{abstract}
RESUMO: Este trabalho teve como objetivo investigar as plantas medicinais utilizadas por idosos assistidos em uma Unidade Básica de Saúde de Pelotas-RS, com diagnóstico de Diabetes mellitus, como terapia complementar no tratamento dos sintomas da doença. Caracterizou-se por pesquisa qualitativa, realizada em julho de 2009, no município de Pelotas-RS. Foram entrevistados 18 idosos com idade entre 60 e 77 anos, sendo 14 do sexo feminino. Os participantes citaram 20 plantas medicinais utilizadas como terapia complementar no tratamento do Diabetes mellitus. Entre estas, as mais citadas utilizadas para diminuir os níveis de glicose no sangue foram Sphagneticola trilobata, Bauhinia spp. e Syzygium cumini, sendo que para as duas últimas há comprovação científica do efeito hipoglicemiante. Ainfusão foi a forma de preparo predominante. Considera-se importante a realização de estudos farmacológicos que investiguem os efeitos das plantas utilizadas pela população, a fim de que o uso proporcione os benefícios desejados e não cause danos à saúde.
\end{abstract}

Palavras-chave: terapias complementares, idoso, enfermagem, doença crônica

\begin{abstract}
Medicinal plants used by elderly people with Diabetes mellitus in the treatment of the disease symptoms. This study aimed to investigate medicinal plants used as complementary therapy by the elderly with diabetes mellitus, assisted by a Health Basic Unit in Pelotas (RS, Brazil). This qualitative research was conducted in July 2009. Eighteen 60 to 77 years old elderly ( 14 of them female) were interviewed. The participants identified 20 medicinal plants used as complementary therapies in the treatment of diabetes mellitus. Among these, the most used ones to reduce the levels of glucose in the blood were Sphagneticola trilobata, Bauhinia spp. and Syzygium cumini. For the last two plants, there are scientific proofs of hypoglycemic effects. The infusion was the predominant form of preparation. Studies to investigate the pharmacological effects of plants used by the population have become very important, for providing scientific information on their possible health benefits and side effects.
\end{abstract}

Key words: complementary therapies, elderly, diabetes, medicinal plants

\section{INTRODUÇÃO}

A utilização de plantas medicinais no tratamento de várias doenças ocorre há milhares de anos. As antigas civilizações já conheciam o poder medicinal de algumas plantas e as cultivavam, repassando os saberes a cada geração. Com o decorrer dos anos e o advento da medicina, este conhecimento passou a ser desvalorizado pelos profissionais de saúde, que começaram a enfocar o tratamento alopático. Porém, atualmente, a ciência e as políticas de saúde estão buscando restabelecer o uso das plantas medicinais pela população.
Com a intenção de implantar a utilização das terapias complementares no Sistema Único de Saúde (SUS) e promover pesquisas com as plantas medicinais, garantindo assim, o uso correto e seguro destas e de fitoterápicos, o Ministério da Saúde (MS) elaborou a Política Nacional de Plantas Medicinais e Fitoterápicos, aprovada pelo Decreto ㄲo 5.813 , de 22 de junho de 2006 (Brasil, 2006a). O MS elaborou a Relação Nacional de Plantas de Interesse ao SUS (RENISUS) em 2009, que apresenta uma lista de 71 plantas medicinais indicadas para uso terapêutico da

Recebido para publicação em 07/12/09

Aceito para publicação em 12/09/11

Rev. Bras. PI. Med., Botucatu, v.14, n.1, p.50-56, 2012. 
população (Brasil, 2009). As plantas medicinais são definidas como espécies vegetais utilizadas para fins terapêuticos que podem ser cultivadas ou não (World Health Organization, 2003).

Por considerar a importância da utilização de plantas no cuidado à saúde pela população, o MS regulamentou a Portaria no 971 em 2006, que aprovou a Política Nacional de Práticas Integrativas e Complementares (PNPIC) no SUS, que indica vários tipos de terapias, dentre as quais a fitoterapia. A fitoterapia é definida como terapêutica caracterizada pelo uso de plantas medicinais em diferentes formas farmacêuticas, sem a utilização de substâncias ativas isoladas (Brasil, 2006b), proporcionando com o uso a normalização das funções fisiológicas do organismo, além de possibilitar interação maior da pessoa com o ambiente (França et al., 2008).

Através deste incentivo do MS e do conhecimento popular, a utilização das plantas no cuidado à saúde está ampliando-se, principalmente entre as pessoas que apresentam alguma doença crônica, como a Diabetes mellitus (DM). Essa é uma doença que ocorre quando o pâncreas não produz insulina em quantidade suficiente ou quando o organismo não consegue utilizar com eficiência a insulina produzida. A hiperglicemia é um efeito comum da doença quando não controlada e, com o passar do tempo, causa lesões graves ao organismo, principalmente aos sistemas nervoso e sanguíneo (World Health Organization, 2009).

Por estes motivos, é necessário estimular a realização de estudos científicos que comprovem o conhecimento popular existente sobre as plantas e a eficácia no tratamento das doenças, evitando assim, os malefícios decorrentes do uso indevido, proporcionando aumento dos benefícios na utilização das plantas medicinais. Assim, este trabalho teve como objetivo investigar as plantas medicinais utilizadas por idosos assistidos em uma Unidade Básica de Saúde de Pelotas-RS, com diagnóstico de Diabetes mellitus, como terapia complementar no tratamento dos sintomas da doença.

\section{MATERIAL E MÉTODO}

Caracterizou-se como um estudo qualitativo, com caráter descritivo. Foi realizado a partir dos dados da pesquisa "Prevalência e fatores associados à depressão em idosos com doenças crônicas" ocorrida no primeiro semestre de 2009, que envolveu idosos com diagnóstico de doenças crônicas prevalentes (Diabetes mellitus - DM, hipertensão arterial sistêmica e/ou doenças musculoesqueléticas) assistidos em uma Unidade Básica de Saúde (UBS), por três equipes da Estratégia de Saúde da Família (ESF), na cidade de Pelotas, localizada na Região Sul do Rio Grande do Sul.
O instrumento utilizado pela pesquisa para a coleta dos dados foi um questionário aplicado aos idosos que haviam frequentado a UBS mais do que duas vezes no último ano e conseguiam comunicarse verbalmente. Este questionário continha questões pré-codificadas, sendo utilizadas para este trabalho as questões que envolviam idosos acometidos por DM, que referiram utilizar plantas medicinais como auxílio no tratamento dos sintomas da doença. $\mathrm{Na}$ pesquisa foram abordados 300 idosos. Destes, 92 $(30,7 \%)$ referiram diagnóstico de DM, sendo que 19 citaram utilizar plantas medicinais.

A partir desta informação, foi aplicado pelas pesquisadoras um questionário semi-estruturado, com questões abertas, em visitas domiciliares agendadas por telefone, aos 18 idosos do estudo. Entre os 19 idosos houve uma recusa, devido à filha negar o diagnóstico da doença e por este motivo relatar não utilizar plantas para esta finalidade. Os dados foram coletados em julho de 2009. Nesta segunda etapa foi realizado o registro fotográfico das plantas medicinais e o georreferenciamento, através do Sistema de Posicionamento Global (GPS). Tevese o cuidado de fotografar o hábito das plantas e, utilizando a função super macro da câmera, fotografar detalhes de ramos, folhas, flores e frutos destas. Não foram produzidas exsicatas das plantas para evitar que o usuário tivesse reduzida disponibilidade de planta para o consumo. A identificação taxonômica foi realizada pelo taxonomista Gustavo Heiden (coautor deste artigo) com base no conjunto de fotografias de cada planta.

Para a análise dos dados, primeiramente as informações foram organizadas e após buscou-se na literatura estudos farmacológicos que comprovassem ou não os efeitos citados pelos entrevistados. Os estudos foram pesquisados em livros científicos e sites na internet (Scientific Electronic Library Online, PubMed, Ministério da Saúde, World Health Organization e International Potato Center).

A pesquisa foi aprovada no Comitê de Ética e Pesquisa da Faculdade de Enfermagem da Universidade Federal de Pelotas sob o parecer 30/ 2009. O termo de consentimento livre e esclarecido foi assinado por todos os participantes, garantindo o anonimato e o direito de não participar ou retirar o consentimento em qualquer momento da pesquisa.

\section{RESULTADO}

Foram entrevistados 18 idosos com idade entre 60 e 77 anos, sendo 14 do sexo feminino. Destes dois tinham diagnóstico de DM tipo 1 e 16 DM tipo 2. Os participantes citaram 20 plantas medicinais (Tabela 1) utilizadas como terapia complementar no tratamento do DM, sendo que de uma não foi possível realizar a identificação taxonômica devido a ausência 
de flores ou frutos. Entre as plantas, as mais citadas para diminuir os níveis de glicose no sangue foram Sphagneticola trilobata, Bauhinia spp. e Syzygium cumini.

A maioria dos idosos (17) utiliza as folhas das plantas, com exceção das plantas Myrrhinium atropurpureum (casca do tronco), Persea americana (caroço) e Polymnia sonchifolia (tubérculo). A infusão foi a forma de preparo citada por 13 entrevistados, três ferviam a planta junto com a água e dois variavam dentre estas duas formas de preparo. Quanto à posologia, variou entre $200 \mathrm{~mL}$ e $2.000 \mathrm{~mL}$ diariamente e $200 \mathrm{~mL}$ a $1.000 \mathrm{~mL}$ por semana.

Citrus sp. (folha) e Persea americana (caroço ralado) são preparados deixando no sereno por sete dias, após esse tempo ingerir uma colher em jejum.

Um dos entrevistados usou Syzygium cumini com dosagem em torno de $200 \mathrm{~mL}$ três vezes ao dia durante um mês e parou, pois constatou que não possuía efeito hipoglicemiante. Já outro relatou que não utiliza há um ano por causar problemas nos olhos e no coração e também devido ao médico de uma amiga ter suspendido o uso.

Entre os locais onde adquirem as plantas, a maioria dos participantes referiu comprar em ervateiros, farmácias e supermercados, coletar na rua, ganhar de vizinhos ou trazer de outras cidades, poucos cultivam as plantas na residência. Os locais de armazenamento são os mais variados, como potes de vidro, plástico ou barro, saco de plástico, caixa de papelão, lata, na geladeira e suspenso na churrasqueira.

Os participantes relataram que o uso das plantas para o diabetes foi indicado por médico, familiares, amigos e pessoas acometidas pela doença que tiveram efeito positivo com a utilização e que

TABELA 1. Plantas medicinais utilizadas pelos idosos como terapia complementar para o Diabetes mellitus. Pelotas, Rio Grande do Sul, 2009.

\begin{tabular}{|c|c|c|c|}
\hline Nome científico & Nome popular & Família & $\begin{array}{c}\text { № de } \\
\text { citações }\end{array}$ \\
\hline \multirow[t]{2}{*}{ Ambrosia sp. } & tripinha-de-galinha, & & \\
\hline & tripa-de-galinha & Asteraceae & 02 \\
\hline Baccharis spp. & carquejinha-branca & Asteraceae & 01 \\
\hline Bauhinia spp. & pata-de-vaca & Fabaceae & 05 \\
\hline Campomanesia aurea Berg & guabiroba & Myrtaceae & 01 \\
\hline Campomanesia xanthocarpa Berg & guabiroba & Myrtaceae & 01 \\
\hline Citrus sp. + Persea americana Mill. & limão + abacate & Rutaceae e Lauraceae & 01 e 01 \\
\hline Cynara scolymus Linn. & alcachofra & Asteraceae & 01 \\
\hline \multicolumn{4}{|l|}{ Echinodorus grandiflorus } \\
\hline (Cham. \& Schlecht.) Micheli & chapéu-de-couro & Alismataceae & 01 \\
\hline Equisetum sp. & cavalinha & Equisetaceae & 01 \\
\hline Hydrangea macrophylla Ser. & hortência & Hidrangeaceae & 01 \\
\hline Morus nigra Linn. & amoreira & Moraceae & 01 \\
\hline Myrrhinium atropurpureum Schott & pau-ferro & Myrtaceae & 01 \\
\hline Phaseolus vulgaris $\mathrm{L}$. & feijão-preto & Fabaceae & 01 \\
\hline Polymnia sonchifolia Poepp. & batata-para-diabetes, & & \\
\hline ou Smallanthus sonchifolius & batata-yacon & Asteraceae & 02 \\
\hline Quercus robur Loudon & carvalho & Fagaceae & 01 \\
\hline Sphagneticola trilobata (L.) Pruski & insulina & Asteraceae & 06 \\
\hline Stachytarpheta cayennensis (Rich.) Vahl & gervão & Verbenaceae & 01 \\
\hline Syzygium cumini (L.) Skeels & jambolão & Myrtaceae & 05 \\
\hline$\left({ }^{*}\right)$ & planta-para-diabetes & - & 01 \\
\hline
\end{tabular}

$\left(^{*}\right)$ Não foi possível realizar a identificação taxonômica da planta devido à ausência de flores e frutos. 
algumas destas não precisaram utilizar medicação. Também obtiveram informação através de reportagens na televisão, revistas de saúde e conversas com outros usuários na UBS. Um idoso referiu que Deus Ihe falou em oração.

A maioria dos idosos (17) faz uso de medicação diariamente. Os medicamentos citados foram Metformina ${ }^{\circledR} 850 \mathrm{mg}$ e $500 \mathrm{mg}$, Glibenclamida ${ }^{\circledR}$ $5 \mathrm{mg}$ e $4 \mathrm{mg}$, Diabnese ${ }^{\circledR} 500 \mathrm{mg}$, Glifage ${ }^{\circledR} 500 \mathrm{mg}$, Glimepirida $^{\circledR} 2$ mg, Galvos ${ }^{\circledR} 50$ mg e insulina NPH ${ }^{\circledR}$.

\section{DISCUSSÃO}

Entre as plantas citadas pelos idosos deste estudo, apenas sete estão presentes na RENISUS (Brasil, 2009): Baccharis trimera, Bauhinia spp. (B. affinis, $B$. forficata ou $B$. variegata), Cynara scolymus, Equisetum arvense, Morus sp., Persea spp. ( $P$. gratissima ou $P$. americana) e Syzygium spp. ( $S$. jambolanum ou S. cumini). A folha foi a parte vegetal mais utilizada da planta, dado similar ao encontrado em outro estudo (Santos et al., 2008).

Encontrou-se para Baccharis genistelloides efeito hipoglicemiante (Xavier et al., 1967) e para Baccharis trimera efeito hepatoprotetor, analgésico, anti-ulcerativo, anti-inflamatório (Soicke et al., 1986; Gene et al., 1996) e potencial atividade anti-diabética (Oliveira et al., 2005), reforçando os efeitos indicados pelo entrevistados.

Bauhinia forficata é amplamente utilizada na medicina popular brasileira para o tratamento de diabetes. Um estudo realizado com ratos diabéticos verificou que o tratamento com esta planta não apresentou efeito hipoglicemiante (Volpato et al., 2008). Em outra pesquisa realizada com extrato aquoso de folhas de $B$. forficata e $C$. sicyoides comprovou-se que apresenta potencial fonte de antioxidantes naturais e pode ser útil na prevenção de complicações diabéticas associadas ao estresse oxidativo (Khalil et al., 2008). O extrato metanólico das folhas de $B$. cheilandra foi testado em ratos diabéticos nas doses de 300, 600 e $900 \mathrm{mg} \mathrm{Kg}^{-1}$, demonstrando atividade hipoglicemiante significativa (Almeida et al., 2006). Foi encontrado também outro estudo que comprova a eficácia de $B$. forficata na redução da hiperglicemia e o mesmo sugere a validade do uso clínico da planta no tratamento do DM tipo 2 (Lino et al., 2004). Estas pesquisas confirmam o uso referido pelos sujeitos do estudo.

Campomanesia xanthocarpa e Cuphea carthagenensis são tradicionalmente usadas no Sul do Brasil na forma de infusão para tratar os níveis elevados de colesterol e triglicérides. $O$ tratamento crônico com extrato aquoso de $C$. xanthocarpa induziu redução significativa no ganho de peso em ratos, em comparação ao grupo controle. Além disso, a análise bioquímica mostrou que esse tratamento reduziu a glicemia, enquanto nenhum efeito sobre os níveis lipídicos foi observado (Biavatti et al., 2004), vindo ao encontro ao efeito citado pelos entrevistados.

Em relação a Cynara scolymus citada pelos indivíduos portadores de Diabetes mellitus, os estudos indicam efeito antioxidante (Skarpanska-Stejnborn et al., 2008), melhora dos sintomas da Síndrome do Intestino Irritável (Bundy et al., 2004) e atividade antiespasmódica (Emendörfer et al., 2005), sem efeitos hipoglicemiantes.

Estudo realizado com o extrato metanólico de Equisetum arvense indicou efeito antidiabético significativo desta planta (Safiyeh et al., 2007). Também foram realizadas pesquisas com as partes aéreas de $E$. myriochaetum, encontrando como resultado efeito hipoglicemiante em pacientes diabéticos tipo 2 a partir de 90 minutos após a administração (Revilla et al., 2002).

Um estudo realizado com Hydrangea macrophylla var. thunbergii em ratos constatou que a planta reduziu significativamente a glicose no sangue, os níveis de triglicérides e de ácidos graxos livres duas semanas após a administração na dose de 200 $\mathrm{mg} \mathrm{kg}^{-1}$ (Zhang et al., 2009).

Com relação a Morus nigra foi encontrado efeito antioxidante moderado (Al-Mustafa \& AlThunibat, 2008) e o fruto da planta tem ação protetora contra danos de biomembranas e biomoléculas (Naderi et al., 2004).

Quanto a Phaseolus vulgaris, através de experimentos com o extrato das vagens em ratos diabéticos foi encontrado efeito hipoglicemiante (Pari \& Venkateswaran, 2003; Pari \& Venkateswaran, 2004) e hipolipemiante (Pari \& Venkateswaran, 2004).

Ao pesquisar sobre Sphagneticola trilobata encontrou-se estudo que trata sobre os potenciais efeitos anti-inflamatórios desta planta (Maldini et al., 2009).

Pesquisa realizada em ratos comprovou o efeito hipoglicemiante de Stachytarpheta cayennensis, com ação similar a glibenclamida (medicação hipoglicemiante) (Adebajo et al., 2007), justificando o uso na busca deste efeito pelos sujeitos do estudo.

Estudo realizado com teste oral de tolerância à glicose mostrou que apenas a casca de Syzygium cumini apresentou atividade anti-hiperglicêmica (Villaseñor \& Lamadrid, 2006). Outro estudo refere que $S$. cumini reduziu a glicemia de ratos nãodiabéticos. Porém, este efeito foi associado com redução do consumo de alimentos e o peso corporal, indicando que este pode não ser um verdadeiro efeito hipoglicemiante (Oliveira et al., 2005). Pesquisas realizadas com as folhas da planta preparadas em forma de chá mostraram que esta não possui efeito hipoglicemiante (Teixeira et al., 2006; Teixeira \& Fuchs, 2006). 
Pesquisa realizada com ratos hipertensos utilizando o extrato bruto etanólico de folhas de Echinodorus grandiflorus demonstrou significativo efeito anti-hipertensivo, sugerindo a eficácia para tratamento oral de hipertensão arterial (Lessa et al., 2008).

Quercus robur demonstrou ter ação antioxidante (Dudonneì et al., 2009), sem efeito na redução da glicemia. $O$ extrato aquoso de Persea americana apresentou redução dos níveis de glicose no sangue de ratos diabéticos (Edem et al., 2009).

A raiz de Polymnia sonchifolia (yacon) apresenta propriedades benéficas ao organismo, entre elas o controle da glicemia (Vilhena et al., 2000). Diferentemente da maioria das raízes, que armazenam carboidratos na forma de amido, o yacon armazena carboidratos na forma de frutanos. O corpo humano não possui enzimas que hidrolisam os fruto-oligossacarídeos, dessa forma, eles passam através do trato digestivo sem ser metabolizados, o que significa que o yacon fornece pouca energia e torna-se alimento promissor para dietas e para os diabéticos, sendo considerado um alimento funcional (Seminario et al., 2003; Candido \& Campos, 2005).

Em relação às plantas identificadas encontrou-se estudos que comprovam a associação com o efeito hipoglicemiante para 11 destas (Baccharis genistelloides, Baccharis trimera, Bauhinia cheilandra, Campomanesia xanthocarpa, Equisetum arvense, Equisetum myriochaetum, Hydrangea macrophylla, Phaseolus vulgaris, Stachytarpheta cayennensis, Persea Americana e Polymnia sonchifolia). Para duas plantas (Bauhinia forficata e Syzygium cumini) os estudos contradizem-se quanto à redução da glicemia.

Para cinco plantas (Cynara scolymus, Morus nigra, Sphagneticola trilobata, Echinodorus grandiflorus e Quercus robur) não foram encontradas investigações que comprovassem o efeito hipoglicemiante destas e para quatro plantas (Ambrosia sp., Campomanesia aurea, Myrrhinium atropurpureum e Citrus sp.) não foram localizadas pesquisas.

Ao analisar o uso das plantas medicinais no cuidado à saúde, destaca-se uma área na qual o enfermeiro pode qualificar-se, devido a esta prática estar sendo estimulada pelo Ministério da Saúde com a introdução das terapias complementares no SUS. Para que isso ocorra, necessita ter conhecimento científico sobre os princípios ativos e contraindicações de cada planta, levando em consideração o conhecimento local, incluindo a diversidade de nomes atribuídos à mesma planta, naquele contexto (Ceolin et al., 2009).

\section{CONCLUSÃO}

Neste estudo identificou-se alguns problemas em relação ao uso das plantas, como a forma de preparo inadequada, a procedência e o armazenamento impróprio, podendo comprometer a qualidade, propriedades funcionais e benefícios à saúde.

É importante destacar que há necessidade de cuidados ao utilizar uma planta medicinal, pois nem todas têm o efeito esperado por quem as utiliza. Isso pode ocorrer devido à forma inadequada de preparo ou ao fato de a planta não possuir o princípio ativo para a ação desejada. Dessa forma, pode-se considerar a importância da realização de estudos farmacológicos que comprovem ou não os efeitos das plantas utilizadas pela população, a fim de que o uso proporcione os benefícios desejados evitando danos à saúde.

Além disso, é fundamental que os profissionais da saúde, em especial os enfermeiros, conheçam o contexto no qual atuam, levando em consideração as plantas utilizadas pela comunidade assistida, buscando capacitar-se nesta área para que possam orientar adequadamente a utilização das plantas medicinais a população.

\section{REFERÊNCIA}

ADEBAJO, A.C. et al. Hypoglycaemic constituents of Stachytarpheta cayennensis leaf. Planta Medica, v.73, n.3, p.241-50, 2007.

ALMEIDA, E.R. et al. Hypoglycemic effect of Bauhinia cheilandra in rats. Fitoterapia, v.77, n.4, p.276-8, 2006.

AL-MUSTAFA, A.H.; AL-THUNIBAT, O.Y. Antioxidant activity of some Jordanian medicinal plants used traditionally for treatment of diabetes. Pakistan Journal Biological Sciences, v.11, n.3, p. 351-8, 2008.

BIAVATTI, M.W. et al. Preliminary studies on Campomanesia xanthocarpa (Berg.) and Cuphea carthagenensis (Jacq.) J.F. Macbr. aqueous extract: weight control and biochemical parameters. Journal of Ethnopharmacology, v.93, n.2-3, p.385-9, 2004.

BUNDY, R. Artichoke leaf extract reduces symptoms of irritable bowel syndrome and improves quality of life in otherwise healthy volunteers suffering from concomitant dyspepsia: a subset analysis. Journal of Alternative and Complementary Medicine, v.10, n.4, p.667-9, 2004.

BRASIL. Ministério da Saúde. Secretaria de Ciência, Tecnologia e Insumos Estratégicos. Departamento de Assistência Farmacêutica. Política Nacional de plantas medicinais e fitoterápicos. Ministério da Saúde, Secretaria de Ciência, Tecnologia e Insumos Estratégicos, Departamento de Assistência Farmacêutica. Brasília: Ministério da Saúde, 2006. 60p. Disponível em: <http:// dtr2004.saude.gov.br/dab/docs/publicacoes/ geral/ pnpmf.pdf>. Acesso em: 05 jul. 2009a.

BRASIL. Ministério da Saúde. Portaria no 971, de 3 de maio de 2006. Política Nacional de Práticas Integrativas e Complementares (PNPIC) no Sistema Único de Saúde. 
2006. 9p. Disponível em: <http://portal.saude.gov.br/portal/ arquivos/pdf/PNPIC.pdf>. Acesso em: 05 jun. 2009b. BRASIL. Ministério da Saúde. Direcção de Administração e Finanças. Secretaria de Ciência, Tecnologia e Insumos Estratégicos. RENISUS - Relação Nacional de Plantas Medicinais de Interesse ao SUS. 2009. 1p. Disponível em: <http://portal.saude.gov.br/ portal/arquivos/pdf/ RENISUS.pdf>. Acesso em: 05 jun. 2009.

CANDIDO, L.M.B.; CAMPOS, A.M. Alimentos funcionais: uma revisão. Ciência e Tecnologia de Alimentos, v.29, n.2, p.193-203, 2005.

CEOLIN, T. et al. Plantas medicinais utilizadas como calmantes por agricultores ecológicos da região Sul do Rio Grande do Sul, Brasil. Revista de Enfermagem UFPE On Line, v.3, n.4, p.253-60, 2009. Disponível em: <http:// www.ufpe.br/revistaenfermagem/ index.php/revista/ article/viewFile/116/116>. Acesso em: 20 nov. 2009.

DUDONNEÉ, S. et al. Comparative study of antioxidant properties and total phenolic content of 30 plant extracts of industrial interest using DPPH, ABTS, FRAP, SOD, and ORAC assays. Journal of Agricultural and Food Chemistry, v.57, n.5, p.1768-74, 2009.

EDEM, D. et al. Effect of aqueous extracts of alligator pear seed (Persea americana Mill) on blood glucose and histopathology of pancreas in alloxan-induced diabetic rats. Pakistan Journal of Pharmaceutical Sciences, v.22, n.3, p.272-6, 2009.

EMENDÖRFER, F. et al. Evaluation of the relaxant action of some Brazilian medicinal plants in isolated guineapig ileum and rat duodenum. Journal of Pharmacy \& Pharmaceutical Sciences, v.8, n.1, p.63-8, 2005.

FRANÇA, I.S.X. et al. Medicina popular: benefícios e malefícios das plantas medicinais. Revista Brasileira de Enfermagem, v.61, n.2, p.201-8, 2008.

GENE, R.M. et al. Anti-inflammatory and analgesic activity of Baccharis trimera: identification of its active constituents. Planta Medica, v.62, n.3, p.232-5, 1996.

KHALIL, N.M. et al. Free radical scavenging profile and myeloperoxidase inhibition of extracts from antidiabetic plants: Bauhinia forficata and Cissus sicyoides. Biological Research, v.41, n.2, p.165-71, 2008.

LESSA, M.A. et al. Antihypertensive effects of crude extracts from leaves of Echinodorus grandiflorus. Fundamental and Clinical Pharmacology, v.22, n.2, p.161-8, 2008.

LINO, C.S. et al. Antidiabetic activity of Bauhinia forficata extracts in alloxan-diabetic rats. Biological and Pharmaceutical Bulletin, v.27, n.1, p.125-7, 2004.

MALDINI, M. et al. Screening of the topical antiinflammatory activity of the bark of Acacia cornigera Willdenow, Byrsonima crassifolia Kunth, Sweetia panamensis Yakovlev and the leaves of Sphagneticola trilobata Hitchcock. Journal of Ethnopharmacology, v.122, n.3, p.430-3, 2009.

NADERI, G.A. et al. Antioxidant activity of three extracts of Morus nigra. Phytotherapy Research, v.18, n.5, p.365-9, 2004.

OLIVEIRA, A.C. et al. Effect of the extracts and fractions of Baccharis trimera and Syzygium cumini on glycaemia of diabetic and non-diabetic mice. Journal of Ethnopharmacology, v.102, n.3, p.465-9, 2005.

PARI, L.; VENKATESWARAN, S. Effect of an aqueous extract of Phaseolus vulgaris on plasma insulin and hepatic key enzymes of glucose metabolism in experimental diabetes. Pharmazie, v.58, n.12, p.916-9, 2003.

PARI, L.; VENKATESWARAN, S. Protective role of Phaseolus vulgaris on changes in the fatty acid composition in experimental diabetes. Journal of Medicinal Food, v.7, n.2, p.204-9, 2004.

REVILLA, M.C. et al. Hypoglycemic effect of Equisetum myriochaetum aerial parts on type 2 diabetic patients. Journal of Ethnopharmacology, v.81, n.1, p.117-20, 2002. SAFIYEH, S. et al. Antidiabetic effect of Equisetum arvense L. (Equisetaceae) in streptozotocin-induced diabetes in male rats. Pakistan Journal of Biological Sciences, v.10, n.10, p.1661-6, 2007.

SANTOS, J.F.L. et al. Uso popular de plantas medicinais na comunidade rural da Vargem Grande, Município de Natividade da Serra, SP. Revista Brasileira de Plantas Medicinais, v.10, n.3, p.67-81, 2008. Disponível em: <http://www.ibb.unesp.br/servicos/ publicacoes/rbpm/ pdf_v10_n3_2008/artigo12_v10n3.pdf>. Acesso em: 20 nov. $200 \overline{9}$.

SEMINARIO, J. et al. El yacón: fundamentos para el aprovechamiento de un recurso promisorio. Centro Internacional de la Papa (CIP), Universidad Nacional de Cajamarca, Agencia Suiza para el Desarrollo y la Cooperación (COSUDE), Lima, Perú, 2003. 60p. Disponível em: <http://www.cipotato.org/artc/docs/ Yacon_Fundamentos_password.pdf>. Acesso em: 20 nov. 2009.

SKARPANSKA-STEJNBORN, A. et al. The influence of supplementation with artichoke (Cynara scolymus L.) extract on selected redox parameters in rowers. International Journal of Sport Nutrition and Exercise Metabolism, v.18, n.3, p.313-27, 2008.

SOICKE, $\mathrm{H}$. et al. Characterization of flavonoids from Baccharis trimera and their antihepatotoxic properties. Planta Medica, v.52, n.1, p.37-9, 1986.

TEIXEIRA, C.C. et al. The efficacy of folk medicines in the management of type 2 diabetes mellitus: results of a randomized controlled trial of Syzygium cumini (L.) Skeels. Journal of Clinical Pharmacy and Therapeutics, v.31, n.1, p.1-5, 2006.

TEIXEIRA, C.C.; FUCHS, F.D. The efficacy of herbal medicines in clinical models: the case of jambolan. Journal of Ethnopharmacology, v.108, n.1, p.16-9, 2006. VILLASEÑOR, I.M.; LAMADRID, M.R. Comparative antihyperglycemic potentials of medicinal plants. Journal of Ethnopharmacology, v.104, n.1-2, p.129-31, 2006.

VILHENA, S.M.C. et al. O cultivo do yacon no Brasil. Horticultura Brasileira, v.189, n.1, p.5-8, 2000.

VOLPATO, G.T. et al. Effect of Bauhinia forficata aqueous extract on the maternal-fetal outcome and oxidative stress biomarkers of streptozotocin-induced diabetic rats. Journal of Ethnopharmacology, v.116, n.1, p.131-7, 2008. XAVIER, A.A. et al. Effect of an extract of Baccharis genistelloides on the glucose level of the blood. Comptes Rendus Sciences de la Societe de Biologie et de ses Filiales, v.161, n.4, p.972-4, 1967.

WORLD HEALTH ORGANIZATION. Guidelines on good agricultural and collection practices (GACP) for medicinal plants. Geneva, 2003. 80p. Disponível em: <http://whqlibdoc.who.int/publications/2003/ 9241546271.pdf>. Acesso em: 10 jul. 2009. 
WORLD HEALTH ORGANIZATION. What is diabetes? 2009. Disponível em: <http://www.who.int/diabetes/en/>. Acesso em: 05 jul. 2009.

ZHANG, $\mathrm{H}$. et al. Hydrangeic acid from the processed leaves of Hydrangea macrophylla var. thunbergii as a new type of anti-diabetic compound. European Journal of Pharmacology, v.606, n.1-3, p.255-61, 2009. 